\title{
Exploring the Infrared Structure of QCD with the Gribov-Zwanziger Lagrangian
}

\author{
J. A. Gracey \\ Theoretical Physics Division, Department of Mathematical Sciences, \\ University of Liverpool, P.O. Box 147, Liverpool, L69 3BX, United Kingdom
}

(Received on 21 July, 2006)

\begin{abstract}
We review recent one and two loop $\overline{\mathrm{MS}}$ Landau gauge calculations using the Gribov-Zwanziger Lagrangian. The behaviour of the gluon and Faddeev-Popov ghost propagators as well as the renormalization group invariant effective coupling constant is examined in the infrared limit.
\end{abstract}

Keywords: QCD; Gribov problem; Confinement

\section{INTRODUCTION}

Quantum Chromodynamics (QCD) is the quantum field theory describing the physics of the strong nuclear force. In the ultraviolet régime the theory describes asymptotically free quarks and gluons, $[1,2]$, which at high energies behave as fundamental particles with propagators of the form $1 / p^{2}$ as the momentum $p$ increases. However, free quarks and gluons are not observed in nature as isolated units due to the confinement mechanism. Moreover, there is a wealth of evidence to indicate that at low energies the behaviour of the gluon propagator differs significantly from that in the ultraviolet. (For example, see the recent reviews of $[3,4]$.) For instance, both Dyson Schwinger equations (DSE) and lattice regularization observe that the gluon propagator vanishes at zero momentum, [5-10]. This property is referred to as gluon suppression. Whilst it has been established in the Landau gauge several other features emerge in the infrared. The propagator of the associated Faddeev-Popov ghost field diverges at zero momentum more rapidly than its $1 / p^{2}$ ultraviolet behaviour. Again this ghost enhancement has been observed in DSE and lattice studies where both approaches obtain good agreement on the value of the exponent in the power law form of the propagator, [5-8]. Another infrared property which is not fully resolved by DSE and lattice analyses is that of the freezing of a particular definition of the effective strong coupling constant. Whilst it is accepted that the particular quantity does not diverge at zero momentum, it is not clear whether it freezes to a finite or zero value. Given that these infrared properties of Landau gauge QCD have been well established by the numerically intensive DSE and lattice regularization techniques, one natural question to pose is, can such phenomena be probed from a Lagrangian point of view, and if not initially quantitatively, can it be accessed and understood qualitatively? This is therefore the purpose of this article, which is primarily to review recent activity in this area, $[11,12]$, by using Zwanziger's elegant study on the Gribov problem, [1316].

Briefly, Gribov pointed out in [17] that one cannot uniquely fix the gauge globally in a non-abelian gauge theory. Specifically for some gauge configurations one can construct one or more copies which can be related by a gauge transformation. To resolve this Gribov proposed that the path integral be restricted to the region defined by the first zero of the FaddeevPopov operator which is defined as the Gribov volume. Whilst one can still have gauge copies inside the Gribov horizon,
[18-20], within it there is a smaller region known as the fundamental modular region where there are no Gribov copies. The key feature of the restriction to the first Gribov region is the introduction of the Gribov mass scale, $\gamma$, which can be related to the volume of the first Gribov region. The restriction of the path integral significantly modifies the behaviour of the gluon and ghost propagators in the infrared, [17]. For instance, the (non-local) Gribov Lagrangian contains a gluon which is infrared suppressed and an enhanced Faddeev-Popov ghost. The latter derives from the one loop ghost 2-point function where the gap equation satisfied by the Gribov mass is central to the one loop enhancement. Whilst Gribov's Landau gauge analysis gave significant insight into the infrared properties, one difficulty was the inability to easily perform practical computations since Gribov's approach effectively amounted to using a Lagrangian with a non-locality. The breakthrough in this area came with Zwanziger's construction of a localised Lagrangian in [13-16]. Significantly that Lagrangian is renormalizable, $[21,22]$, and therefore allows one to carry out calculations.

\section{GRIBOV-ZWANZIGER LAGRANGIAN}

The Gribov-Zwanziger Lagrangian involves two pieces, [14]. One is the usual Landau gauge fixed QCD Lagrangian and the other part involves additional fields which we will refer to as Zwanziger ghosts. These are $\left\{\phi_{\mu}^{a b}, \bar{\phi}_{\mu}^{a b}, \omega_{\mu}^{a b}, \bar{\omega}_{\mu}^{a b}\right\}$ where the latter pair are anticommuting. The full localised renormalizable Lagrangian is, [14],

$$
\begin{aligned}
L^{G Z}= & L^{Q C D}+\bar{\phi}^{a b \mu} \partial^{\nu}\left(D_{\nu} \phi_{\mu}\right)^{a b}-\bar{\omega}^{a b \mu} \partial^{\nu}\left(D_{\nu} \omega_{\mu}\right)^{a b} \\
& -g f^{a b c} \partial^{v} \bar{\omega}_{\mu}^{a e}\left(D_{\nu} c\right)^{b} \phi^{e c \mu} \\
& +\frac{\gamma^{2}}{\sqrt{2}}\left(f^{a b c} A^{a \mu} \phi_{\mu}^{b c}+f^{a b c} A^{a \mu} \bar{\phi}_{\mu}^{b c}\right)-\frac{d N_{A} \gamma^{4}}{2 g^{2}}
\end{aligned}
$$

where $L^{Q C D}$ denotes the usual Lagrangian involving the gluon $A_{\mu}^{a}$ and Faddeev-Popov ghost fields $c^{a}$ and $\bar{c}^{a}, D_{\mu}$ is the usual covariant derivative, $f^{a b c}$ are the colour group structure functions, $N_{A}$ is the dimension of the adjoint representation, $g$ is the coupling constant, and $d$ is the spacetime dimension. The commuting Zwanziger ghosts $\phi_{\mu}^{a b}$ and $\bar{\phi}_{\mu}^{a b}$ implement the Gribov horizon condition which in the Gribov formulation is defined by the vacuum expectation value, [17],

$$
\left\langle A_{\mu}^{a}(x) \frac{1}{\partial^{v} D_{v}} A^{a \mu}(x)\right\rangle=\frac{d N_{A}}{C_{A} g^{2}} .
$$


The equivalent definition in (1) is, [14],

$$
f^{a b c}\left\langle A^{a \mu}(x) \phi_{\mu}^{b c}(x)\right\rangle=\frac{d N_{A} \gamma^{2}}{\sqrt{2} g^{2}}
$$

and these two equations define the gap equation satisfied by the Gribov mass $\gamma$. The relation between both definitions of the horizon conditions is made manifest from the equation of motion of the Zwanziger ghost

$$
\phi_{\mu}^{a b}=\frac{\gamma^{2}}{\sqrt{2}} f^{a b c} \frac{1}{\partial^{v} D_{v}} A_{\mu}^{c} .
$$

Further, focusing on the part of the Lagrangian involving purely gluons and using (4) then the non-locality of the original formulation of Gribov becomes apparent

$$
L=-\frac{1}{4} G_{\mu \nu}^{a} G^{a \mu \nu}+\frac{C_{A} \gamma^{4}}{2} A_{\mu}^{a} \frac{1}{\partial^{v} D_{v}} A^{a \mu}-\frac{d N_{A} \gamma^{4}}{2 g^{2}} .
$$

As with any Lagrangian one needs to determine the explicit values of the renormalization constants and hence anomalous dimensions in order to perform practical calculations. As was demonstrated in $[21,22]$ the Landau gauge Gribov-Zwanziger Lagrangian has an interesting renormalization structure. In addition to the renormalization constants of the usual QCD Lagrangian the new fields and parameters each have a renormalization constant

$$
\phi_{\mathrm{O}}^{a b \mu}=\sqrt{Z_{\phi}} \phi^{a b \mu}, \omega_{\mathrm{O}}^{a b \mu}=\sqrt{Z_{\omega}} \omega^{a b \mu}, \gamma_{\mathrm{o}}=Z_{\gamma} \gamma
$$

where the subscript o denotes the bare quantity. However, it was demonstrated in $[21,22]$ by the algebraic renormalization programme that to all orders in perturbation theory in the Landau gauge

$$
Z_{c}=Z_{\phi}=Z_{\omega}
$$

and

$$
Z_{\gamma}=Z_{A}^{1 / 4} Z_{c}^{1 / 4}
$$

where $Z_{A}$ and $Z_{c}$ are respectively the gluon and FaddeevPopov ghost renormalization constants. Hence, no new renormalization constants are required and, moreover, the original QCD anomalous dimensions for the gluon, Faddeev-Popov ghost and quark as well as the $\beta$-function remain unchanged. So the presence of the localising fields does not alter the ultraviolet running. For completeness we note that the anomalous dimension of $\gamma$ is known to three loops in $\overline{\mathrm{MS}}$ for arbitrary colour group and at four loops for $S U\left(N_{c}\right)$ in the Landau gauge, [23].

Whilst this article primarily deals with the Landau gauge, as an aside one can begin to address what happens in other linear covariant gauges aside from the Landau gauge by restoring the usual $\alpha$ dependent part to $L^{Q C D}$ in (1). In [24] the construction of the horizon function has been examined for small $\alpha$ where $\alpha$ is the gauge fixing parameter in a linear covariant gauge. In this approximation one can repeat some of the Landau gauge construction and establish a gap equation.
In this context one can examine the renormalization of (1) where $L^{Q C D}$ is replaced by the $\alpha$ dependent Lagrangian. It is a straightforward exercise to examine the three loop renormalization for non-zero $\alpha$ which has not been determined before. The machinery to do this involves the application of the MINCER algorithm, [25, 26], written in the symbolic manipulation language FORM, [27], and the intensive use of computer algebra. The full three loop renormalization of the $\alpha$ extended Gribov-Zwanziger Lagrangian has been performed in the $\overline{\mathrm{MS}}$ scheme and the following $\alpha$ dependent anomalous dimensions emerged for the additional fields and $\gamma$ to three loops

$$
\begin{aligned}
\gamma_{c}(a)= & \gamma_{\phi}(a)=\gamma_{\omega}(a) \\
\gamma_{\gamma}(a)= & {\left[16 T_{F} N_{f}-(35+3 \alpha) C_{A}\right] \frac{a}{48} } \\
& +\left[(3 \alpha-449) C_{A}^{2}+280 T_{F} N_{f} C_{A}+192 T_{F} N_{f} C_{A}\right] \frac{a}{192} \\
& +\left[(1512 \alpha-15552 \zeta(3)+89008) T_{F} N_{f} C_{A}^{2}\right. \\
& -3456 T_{F} N_{f} C_{F}^{2}-8448 T_{F}^{2} N_{f}^{2} C_{F}-12352 T_{F}^{2} N_{f}^{2} C_{A} \\
& +(20736 \zeta(3)+19920) T_{F} N_{f} C_{A} C_{F} \\
& -\left(81 \alpha^{3}-162 \zeta(3) \alpha^{2}+162 \alpha^{2}-648 \zeta(3) \alpha+918 \alpha\right. \\
& \left.-486 \zeta(3)+75607) C_{A}^{3}\right] \frac{a^{3}}{6912}+O\left(a^{4}\right)
\end{aligned}
$$

where $a=g^{2} /\left(16 \pi^{2}\right), \zeta(n)$ is the Riemann zeta function and the group Casimirs are defined by

$$
T^{a} T^{a}=C_{F} I, f^{a c d} f^{b c d}=C_{A} \delta^{a b}, \operatorname{Tr}\left(T^{a} T^{b}\right)=T_{F} \delta^{a b}
$$

where $T^{a}$ are the generators of the Lie algebra of the colour group. Throughout we use dimensional regularization in $d=4-2 \varepsilon$ dimensions. To establish $\gamma_{\gamma}(a)$ we have used the version of the Gribov-Zwanziger Lagrangian where the $\gamma$ dependent terms are regarded as being part of the interaction Lagrangian. Therefore, the gluon and $\phi_{\mu}^{a b}$ fields are regarded as massless. This is the form required for applying the MINCER algorithm which determines massless 2-point functions to three loops and the finite part in $\varepsilon$. Thus $\gamma_{\gamma}(a)$ is determined by renormalizing the Green's function where the operator associated with $\gamma^{2}$ in (1) is inserted at zero momentum into the $A_{\mu}^{a}-\phi_{v}^{b c}$ Green's function. This involved 2 one loop, 43 two loop and 1082 three loop Feynman diagrams which were generated with the QGRAF package, [28].

Clearly the first Slavnov-Taylor identity, (7), appears to hold for all $\alpha$ and is central to the observation that like the Landau gauge the other anomalous dimensions (and the $\beta$ function) are equivalent to their $\alpha$ dependent values with or without the localising features. By contrast the Gribov mass parameter is not only $\alpha$ dependent but is not proportional to the sum of the gluon and Faddeev-Popov ghost anomalous dimensions as was the case in the Landau gauge. Therefore, for non-zero $\alpha$ an extra independent renormalization constant is required to render (1) finite unlike the Landau gauge. 


\section{GAP EQUATION}

Having established the Gribov-Zwanziger Lagrangian as a tool to perform calculations one can begin to address the problem of what its properties are at zero momentum, returning to the Landau gauge. First, one can construct the gap equation satisfied by $\gamma$ at two loops by evaluating the vacuum expectation value (3). This requires the Feynman rules of (1) as well as the propagators. Due to the mixed 2-point term in (1) the $A_{\mu}^{a}$ and $\phi_{\mu}^{a b}$ sector propagators are

$$
\begin{aligned}
\left\langle A_{\mu}^{a}(p) A_{v}^{b}(-p)\right\rangle= & -\frac{\delta^{a b} p^{2}}{\left[\left(p^{2}\right)^{2}+C_{A} \gamma^{4}\right]} P_{\mu v}(p) \\
\left\langle A_{\mu}^{a}(p) \bar{\phi}_{v}^{b c}(-p)\right\rangle= & -\frac{f^{a b c} \gamma^{2}}{\sqrt{2}\left[\left(p^{2}\right)^{2}+C_{A} \gamma^{4}\right]} P_{\mu v}(p) \\
\left\langle\phi_{\mu}^{a b}(p) \bar{\phi}_{v}^{c d}(-p)\right\rangle= & -\frac{\delta^{a c} \delta^{b d}}{p^{2}} \eta_{\mu \nu} \\
& +\frac{f^{a b e} f^{c d e} \gamma^{4}}{p^{2}\left[\left(p^{2}\right)^{2}+C_{A} \gamma^{4}\right]} P_{\mu v}(p)
\end{aligned}
$$

where $P_{\mu v}(p)=\eta_{\mu v}-p_{\mu} p_{v} / p^{2}$. Therefore using these to evaluate the 1 one loop and 17 two loop Feynman graphs contributing to (3), we arrive at the two loop $\overline{\mathrm{MS}}$ gap equation satisfied by the running Gribov mass $\gamma$, for $N_{f}$ massless quarks, [11],

$$
\begin{aligned}
1= & C_{A}\left[\frac{5}{8}-\frac{3}{8} \ln \left(\frac{C_{A} \gamma^{4}}{\mu^{4}}\right)\right] a \\
+ & {\left[C _ { A } ^ { 2 } \left(\frac{2017}{768}-\frac{11097}{2048} s_{2}+\frac{95}{256} \zeta(2)-\frac{65}{48} \ln \left(\frac{C_{A} \gamma^{4}}{\mu^{4}}\right)\right.\right.} \\
& \left.+\frac{35}{128}\left(\ln \left(\frac{C_{A} \gamma^{4}}{\mu^{4}}\right)\right)^{2}+\frac{1137}{2560} \sqrt{5} \zeta(2)-\frac{205 \pi^{2}}{512}\right) \\
& +C_{A} T_{F} N_{f}\left(-\frac{25}{24}-\zeta(2)+\frac{7}{12} \ln \left(\frac{C_{A} \gamma^{4}}{\mu^{4}}\right)\right. \\
& \left.\left.-\frac{1}{8}\left(\ln \left(\frac{C_{A} \gamma^{4}}{\mu^{4}}\right)\right)^{2}+\frac{\pi^{2}}{8}\right)\right] a^{2}+O\left(a^{3}\right)
\end{aligned}
$$

where $s_{2}=(2 \sqrt{3} / 9) \mathrm{Cl}_{2}(2 \pi / 3)$ with $\mathrm{Cl}_{2}(x)$ the Clausen function and $\mu$ is the scale introduced in dimensional regularization which ensures a dimensionless coupling constant in $d$ dimensions. This calculation also required the use of the QGRAF package, [28], to generate the electronic version of the Feynman diagrams as well as the use of FORM to handle the algebra. Additionally the two loop master vacuum bubble integral

$$
I\left(m_{1}^{2}, m_{2}^{2}, m_{3}^{2}\right)=\int_{k l} \frac{1}{\left[k^{2}-m_{1}^{2}\right]\left[l^{2}-m_{2}^{2}\right]\left[(k-l)^{2}-m_{3}^{2}\right]}
$$

where $m_{i}^{2} \in\left\{0, i \sqrt{C_{A}} \gamma^{2},-i \sqrt{C_{A}} \gamma^{2}\right\}$ was required to the finite part in $\varepsilon$.

\section{INFRARED PROPERTIES}

As Gribov was able to establish the one loop FaddeevPopov ghost enhancement by evaluating the ghost 2-point function and applying the one loop gap equation, [17], it is now viable to extend that calculation. At two loops there are 31 Feynman diagrams to evaluate. If we write the FaddeevPopov form factor as

$$
D_{c}\left(p^{2}\right)=\frac{1}{\left[1+u\left(p^{2}\right)\right]}
$$

where $D_{c}\left(p^{2}\right) / p^{2}$ represents the propagator itself, then $u\left(p^{2}\right)$ represents the radiative corrections. So ghost enhancement then translates into the Kugo-Ojima confinement criterion of $u(0)=-1$, [29]. Hence, to consider this in the GribovZwanziger context one needs to evaluate the two loop radiative corrections in the small $p^{2}$ expansion. In practical terms this means carrying out the vacuum bubble expansion of the Feynman diagrams and using the master integral (13), for instance, and the cases where the propagators have integer powers larger than unity. Completing this one finds that $u(0)=-1$ at $t w o$ loops precisely when $\gamma$ satisfies the gap equation of (12), [11]. In extending the one loop result of Gribov to two loops means that the Kugo-Ojima confinement criterion is consistent at two loops in (1) as well as being a check on the actual explicit computation of (12). Moreover, the fact that the condition is precisely satisfied at two loops is also consistent with the all orders proof given in [15]. There general arguments established that the ghost propagator behaves as $1 /\left(p^{2}\right)^{2}$ as $p^{2} \rightarrow 0$ in (1).

Repeating the analysis for the gluon propagator is more involved due to the mixing of $A_{\mu}^{a}$ with $\phi_{\mu}^{a b}$ in the quadratic part of the Lagrangian. If one formally writes the one loop correction to the $2 \times 2$ matrix of 2-point functions as, [12],

$$
\left(\begin{array}{cc}
p^{2} \delta^{a c} & -\gamma^{2} f^{a c d} \\
-\gamma^{2} f^{c a b} & -p^{2} \delta^{a c} \delta^{b d}
\end{array}\right)+\left(\begin{array}{cc}
X \delta^{a c} & U f^{a c d} \\
M f^{c a b} & N^{a b c d}
\end{array}\right) a+O\left(a^{2}\right)
$$

where the common factor of $P_{\mu v}(p)$ in the Landau gauge has been factored off, $N^{a b c d}=Q \delta^{a c} \delta^{b d}+W f^{a c e} f^{b d e}+R f^{a b e} f^{c d e}$ $+S d_{A}^{a b c d}$ and

$$
d_{A}^{a b c d}=\operatorname{Tr}\left(T_{A}^{(a} T_{A}^{b} T_{A}^{c} T_{A}^{d)}\right)
$$

where $T_{A}^{a}$ is the colour group generator in the adjoint representation. The 14 one loop Feynman graphs contributing to the 2-point function matrix have been evaluated both exactly and in the vacuum bubble expansion and are in agreement when the exact expression is expanded in powers of $p^{2}$. Inverting the 2-point matrix gives the propagator matrix and formally we find the propagators to one loop are given by, [12],

$$
\begin{aligned}
& \left(\begin{array}{cc}
\frac{p^{2}}{\left[\left(p^{2}\right)^{2}+C_{A} \gamma^{4}\right]} \delta^{c p} & -\frac{\gamma^{2}}{\left[\left(p^{2}\right)^{2}+C_{A} \gamma^{4}\right]} f^{c p q} \\
-\frac{\gamma^{2}}{\left[\left(p^{2}\right)^{2}+C_{A} \gamma^{4}\right]} f^{p c d} & -\frac{1}{p^{2}} \delta^{c p} \delta^{d q}+\frac{\gamma^{4} f^{c d r} f^{p q r}}{p^{2}\left[\left(p^{2}\right)^{2}+C_{A} \gamma^{4}\right]}
\end{array}\right) \\
+ & \left(\begin{array}{cc}
A \delta^{c p} & C f^{c p q} \\
E f^{p c d} & \Phi^{c d p q}
\end{array}\right) a+O\left(a^{2}\right)
\end{aligned}
$$


where

$$
\Phi^{c d p q}=G \delta^{c p} \delta^{d q}+J f^{c p e} f^{d q e}+K f^{c d e} f^{p q e}+L d_{A}^{c d p q}
$$

and

$$
\begin{array}{r}
A=\frac{(-1)}{\left[\left(p^{2}\right)^{2}+C_{A} \gamma^{4}\right]^{2}}\left[\left(p^{2}\right)^{2} X-2 C_{A} \gamma^{2} p^{2} U\right. \\
\left.+C_{A} \gamma^{4}\left(Q+C_{A} R+\frac{1}{2} C_{A} W\right)\right] .
\end{array}
$$

The first entry in this matrix corresponds to the correction to the gluon propagator and in terms of the 2-point functions is

$$
\begin{array}{r}
A=\frac{(-1)}{\left[\left(p^{2}\right)^{2}+C_{A} \gamma^{4}\right]^{2}}\left[\left(p^{2}\right)^{2} X-2 C_{A} \gamma^{2} p^{2} U\right. \\
\left.+C_{A} \gamma^{4}\left(Q+C_{A} R+\frac{1}{2} C_{A} W\right)\right] .
\end{array}
$$

Hence, if one writes the form factor of the gluon as $D_{A}\left(p^{2}\right)$ where $D_{A}\left(p^{2}\right) P_{\mu v}(p) / p^{2}$ is the propagator, then one finds that $D_{A}\left(p^{2}\right) \rightarrow 0$ as $p^{2} \rightarrow 0$ at one loop, [12]. Hence, one has gluon suppression at this order in (1) consistent with DSE and lattice studies.

We close this section on the propagators by noting that for the other fields one and two loop calculations reveal parallel behaviour. For the $\omega_{\mu}^{a b}$ field it too has a completely similar enhancement at two loops if one computes the two loop 2point function, in a similar way to that of the Faddeev-Popov ghost, and applies the two loop gap equation. So the KugoOjima confinement criterion is satisfied by both sets of anticommuting fields. However, it is worth noting that the original Kugo-Ojima analysis was for a non-abelian gauge theory with only Faddeev-Popov ghosts and not with the additional Zwanziger ghosts. Therefore, for completeness it would seem that the study of [29] needs to be extended to the case of (1). Equally at one loop the exact expressions for both the $\omega_{\mu}^{a b}$ and Faddeev-Popov ghost form factors are the same. Explicitly we have

$$
\begin{aligned}
& D_{c}\left(p^{2}\right)=D_{\omega}\left(p^{2}\right) \\
& =\left[\left[\frac{5}{8}+\frac{\pi p^{2}}{8 \sqrt{C_{A}} \gamma^{2}}+\frac{3 \sqrt{C_{A}} \gamma^{2}}{4 p^{2}} \tan ^{-1}\left[\frac{\sqrt{C_{A}} \gamma^{2}}{p^{2}}\right]\right.\right. \\
& \quad-\frac{3}{8} \ln \left[1+\frac{\left(p^{2}\right)^{2}}{C_{A} \gamma^{4}}\right]+\frac{C_{A} \gamma^{4}}{8\left(p^{2}\right)^{2}} \ln \left[1+\frac{\left(p^{2}\right)^{2}}{C_{A} \gamma^{4}}\right] \\
& \left.\left.\quad-\frac{3 \pi \sqrt{C_{A}} \gamma^{2}}{8 p^{2}}-\frac{p^{2}}{4 \sqrt{C_{A}} \gamma^{2}} \tan ^{-1}\left[\frac{\sqrt{C_{A}} \gamma^{2}}{p^{2}}\right]\right] C_{A} a\right]^{-1} .
\end{aligned}
$$

Moreover, these facts suggest that there is an all orders equality which, if so, could possibly be established by general symmetry arguments. Also, whilst the $\phi_{\mu}^{a b} 2$-point function is more complicated due to the appearance of the colour group tensors, the channel corresponding to the tree term in the Lagrangian is equivalent at one loop in $\overline{\mathrm{MS}}$ to the gap equation. This suggests that in this channel there is a change in its infrared behaviour.

\section{EFFECTIVE COUPLING CONSTANT}

One quantity which receives wide attention in both DSE and lattice studies is the renormalization group invariant definition of the effective strong coupling constant based on the renormalization properties of the ghost gluon vertex. (See, for instance, [4].) Since this vertex does not undergo renormalization in the Landau gauge, then the effective coupling constant is defined from the gluon and ghost form factors as

$$
\alpha_{S}^{\mathrm{eff}}\left(p^{2}\right)=\alpha(\mu) D_{A}\left(p^{2}\right)\left(D_{c}\left(p^{2}\right)\right)^{2}
$$

where $\alpha(\mu)=g^{2}(\mu) /(4 \pi)$ is the running strong coupling constant. Although both numerical approaches use the same definition they do not definitively agree on the infrared behaviour aside from the fact that there is freezing at a finite value. However, some DSE studies find $\alpha_{S}^{\text {eff }}(0)=2.97$ for $S U(3),[30,31]$, whilst various lattice and DSE analyses suggest $\alpha_{S}^{\text {eff }}(0)=0,[32,33]$. Given that we have analysed both form factors in the Gribov-Zwanziger Lagrangian, it is a simple exercise to evaluate (22) in the $p^{2} \rightarrow 0$ limit. We find that at one loop in the $\overline{\mathrm{MS}}$ scheme, [12],

$$
\alpha_{S}^{\mathrm{eff}}(0)=\lim _{p^{2} \rightarrow 0}\left[\frac{\alpha(\mu)\left[1+C_{A}\left(\frac{3}{8} L-\frac{215}{384}\right) a\right]\left(p^{2}\right)^{2}}{C_{A} \gamma^{4}\left[1+C_{A}\left(\frac{3}{8} L-\frac{5}{8}+\frac{\pi p^{2}}{8 \sqrt{C_{A}} \gamma^{2}}\right) a\right]^{2}}\right]
$$

where $L=\ln \left(\frac{C_{A} \gamma^{4}}{\mu^{4}}\right)$. Thus for $S U(3)$ we find $\alpha_{S}^{\text {eff }}(0)=1.768$ independent of the number of massless quarks. Whilst this is less than the value for the DSE approach it is finite and nonzero. Moreover, the $N_{c}$ dependence is in qualitative agreement with $[30,31]$. In the determination of a finite value it is worth noting the key role played by the gap equation in ensuring the cancellation of the dimensionful and dimensionless quantities to leave a pure number. We also remark that the $\omega_{\mu}^{a b}$ gluon vertex has a similar freezing to the same value, [12].

One property of (22) which motivated recent studies of Landau gauge QCD and the suggested condensation of low dimensional operators was the numerical evidence of a $1 / p^{2}$ power correction to the effective coupling constant, [34-37]. Although that analysis was in the $\overline{\mathrm{MOM}}$ scheme, if it is a more general property of the Landau gauge effective coupling constant it ought to be present in other schemes. As we have exact expressions for the form factors it is possible to evaluate (22) in powers of $p^{2}$ and see whether a $1 / p^{2}$ term emerges as a correction or if the expected $1 /\left(p^{2}\right)^{2}$ term is the first term. The latter is certainly expected to be present due to the existence of the gauge invariant dimension four operator $\left(G_{\mu \nu}^{a}\right)^{2}$ which can condense. Therefore, returning to (15) and formally expanding in powers of $\gamma^{2}$, we have at leading order at one loop, 
[12],

$$
\begin{aligned}
X= & {\left[\left[\left(\frac{13}{6} \ln \left(\frac{p^{2}}{\mu^{2}}\right)-\frac{97}{36}\right) p^{2}+\frac{3 \pi \sqrt{C_{A}} \gamma^{2}}{8}\right] C_{A}\right.} \\
& \left.-\left[\frac{4}{3} \ln \left(\frac{p^{2}}{\mu^{2}}\right)-\frac{20}{9}\right] T_{F} N_{f} p^{2}\right] a \\
M= & U=\left[\frac{11 C_{A}}{8} \gamma^{2}\right] a \\
Q= & {\left[\left[-\left(1-\frac{3}{4} \ln \left(\frac{p^{2}}{\mu^{2}}\right)\right) p^{2}+\frac{3 \pi \sqrt{C_{A}} \gamma^{2}}{8}\right] C_{A}\right] a } \\
W= & R=S=O\left(a^{2}\right) .
\end{aligned}
$$

Hence, at one loop

$$
\begin{aligned}
& D_{A}\left(p^{2}\right)=1-\frac{3 C_{A} \pi}{8} \frac{\sqrt{C_{A}} \gamma^{2}}{p^{2}} a \\
& D_{c}\left(p^{2}\right)=\left[-1+\left[1-\frac{3}{4} \ln \left(\frac{p^{2}}{\mu^{2}}\right)-\frac{3 \pi}{8} \frac{\sqrt{C_{A}} \gamma^{2}}{p^{2}}\right] C_{A} a\right]^{-1} .
\end{aligned}
$$

Thus evaluating $\alpha_{S}^{\text {eff }}\left(p^{2}\right)$ in the same expansion and identifying the $\gamma$ independent pieces as deriving from the perturbative part of the coupling constant, we find at one loop

$$
\alpha_{S}^{\text {eff }}\left(p^{2}\right)=\alpha_{S}^{\text {pert }}\left(p^{2}\right)\left[1-\frac{9 C_{A} \pi}{8} \frac{\sqrt{C_{A}} \gamma^{2}}{p^{2}} a+O\left(\frac{1}{\left(p^{2}\right)^{2}}\right)\right] .
$$

Thus a $1 / p^{2}$ correction emerges and the power correction of [34-37] can be mimicked by the presence of the Gribov mass in the Gribov-Zwanziger Lagrangian. Though this ought to be qualified by noting that the condensation of the gauge variant operator $\frac{1}{2}\left(A_{\mu}^{a}\right)^{2}$ has also received a large amount of attention in recent years in the Landau gauge, $[38,39]$, and could be an alternative justification for the lattice result. It is also worth observing that both $\gamma^{2}$ and $\frac{1}{2}\left(A_{\mu}^{a}\right)^{2}$ have the same anomalous dimensions in the Landau gauge $[40,41]$. Indeed the effect that the Gribov mass has on the condensation of $\frac{1}{2}\left(A_{\mu}^{a}\right)^{2}$ has been examined in $[22,42,43]$. One final point is that the sign of $\gamma^{2}$ is not fixed in (1). Therefore, one can alter the sign of $\gamma^{2}$ in (26) to obtain the same sign as that in [36,37]. Although this is for a different scheme ( $\overline{\mathrm{MOM}})$ compared to our $\overline{\mathrm{MS}}$ result, it would result in an effective tachyonic gluon mass. This may appear unacceptable. However, as the gluon is not truly an observable field due to confinement this ought not to be a hindrance. More specifically this tachyonic nature is only revealed in the next to high energy limit. If one examines the full gluon propagator at one loop, there is no pole in the propagator. Therefore, one cannot truly identify a mass for the confined gluon in the conventional way that one does for a fundamental unconfined field. Another observation from a phenomenological point of view is that in [44] it was noted that a tachyonic gluon mass in current correlators could reproduce experimental data more accurately than either a massless gluon or one with a non-tachyonic mass.

\section{CONCLUSION}

We have studied the Gribov-Zwanziger Lagrangian, [14], at one loop and established that it qualitatively reproduces the propagator and effective coupling constant behaviour observed in the infrared using DSE and lattice studies. Moreover, the gap equation has been determined at two loops in $\overline{\mathrm{MS}}$ and ensures the Kugo-Ojima confinement criterion is satisfied. This would appear to establish the Gribov-Zwanziger Lagrangian as a useful tool to examine other phenomenological aspects of QCD such as the operator product expansion and the evaluation of vacuum expectation values of gauge invariant operators, such as $\left(G_{\mu v}^{a}\right)^{2}$. Further, we have examined the renormalization of the Gribov-Zwanziger Lagrangian in linear covariant gauges albeit in the limit of small gauge fixing parameter. Clearly it would be a formidable task to fully extend the Landau gauge Gribov-Zwanziger Lagrangian to other gauges such as linear covariant and maximal abelian gauges, $[24,45]$. However, if it were possible then it would be interesting to extend our present Landau gauge propagator and effective coupling constant analysis to these gauges in order to understand what their infrared structure is. This would complement DSE and lattice analyses.

\section{ACKNOWLEDGEMENTS}

The author thanks Prof. S. Sorella, Prof. D. Zwanziger, and Dr D.Dudal for useful discussions concerning the Gribov problem.
[1] D.J. Gross and F.J. Wilczek, Phys. Rev. Lett. 30, 1343 (1973).

[2] H.D. Politzer, Phys. Rev. Lett. 30, 1346 (1973).

[3] R. Alkofer and L. von Smekal, Phys. Rep. 353, 281 (2001).

[4] C. S. Fischer, J. Phys. G 32, R253 (2006).

[5] L. von Smekal, A. Hauck, and R. Alkofer, Phys. Rev. Lett. 79, 3591 (1997).

[6] L. von Smekal, A. Hauck, and R. Alkofer, Annals Phys. 267, 1 (1998).

[7] D. Zwanziger, Phys. Rev. D 65, 094039 (2002).

[8] C. Lerche and L. von Smekal, Phys. Rev. D 65, 125006 (2002).

[9] P. Boucaud, T. Brüntjen, J.P. Leroy, A. Le Yaounac, A.Y.
Lokhov, J. Micheli, O. Pène, J. Rodríguez-Quintero, and C. Roiesnel, hep-lat/0602006.

[10] E.M. Ilgenfritz, M. Müller-Preussker, A. Sternbeck, and A. Schiller, hep-lat/0601027.

[11] J.A. Gracey, Phys. Lett. B 632, 282 (2006)

[12] J.A. Gracey, JHEP 0605, 052 (2006).

[13] D. Zwanziger, Nucl. Phys. B 321, 591 (1989).

[14] D. Zwanziger, Nucl. Phys. B 323, 513 (1989).

[15] D. Zwanziger, Nucl. Phys. B 399, 477 (1993).

[16] D. Zwanziger, Nucl. Phys. B 378, 525 (1992).

[17] V.N. Gribov, Nucl. Phys. B 139, 1 (1978). 
[18] Semenov-Tyan-Shanskii and V.A. Franke, Zapiski Nauchnykh Seminarov Leningradskogo Otdeleniya Matematsicheskogo Instituta im. V.A. Steklov AN SSSR 120 (1982) 159. English translation: New York: Plenum Press 1986.

[19] G. Dell'Antonio and D. Zwanziger, Commun. Math. Phys. 138, 291 (1991).

[20] P. van Baal, Nucl. Phys. B 369, 259 (1992).

[21] N. Maggiore and M. Schaden, Phys. Rev. D 50, 6616 (1994).

[22] D. Dudal, R.F. Sobreiro, S.P. Sorella, and H. Verschelde, Phys. Rev. D 72, 014016 (2005).

[23] K.G. Chetyrkin, Nucl. Phys. B 710, 499 (2005).

[24] R.F. Sobreiro \& S.P. Sorella, JHEP 06, 054 (2005).

[25] S.G. Gorishny, S.A. Larin, L.R. Surguladze, and F.K. Tkachov, Comput. Phys. Commun. 55, 381 (1989).

[26] S.A. Larin, F.V. Tkachov, and J.A.M. Vermaseren, "The Form version of Mincer", NIKHEF-H-91-18.

[27] J.A.M. Vermaseren, math-ph/0010025.

[28] P. Nogueira, J. Comput. Phys. 105, 279 (1993).

[29] T. Kugo and I. Ojima, Prog. Theor. Phys. Suppl. 66, 1 (1979); T. Kugo and I. Ojima, Prog. Theor. Phys. Suppl. 77, 1121 (1984).

[30] R. Alkofer, C.S. Fischer, and F.J. Llanes-Estrada, Phys. Lett. B 611, 279 (2005).

[31] C.S. Fischer, PoS LAT2005 (2005), 330.

[32] A.C. Aguilar \& A.A. Natale, JHEP 08, 057 (2004).

[33] A. Sternbeck, E.M. Ilgenfritz, M. Müller-Preussker \& A. Schiller, Nucl. Phys. Proc. Suppl. 153, 185 (2006).
[34] G. Burgio, F. di Renzo, G. Marchesini, and E. Onofri, Phys. Lett. B 422, 219 (1998).

[35] P. Boucaud, G. Burgio, F. di Renzo, J.P. Leroy, J. Micheli, C. Parrinello, O. Pène, C. Pittori, J. Rodriguez-Quintero, C. Roiesnel, and K. Sharkey, JHEP 04, 006 (2000).

[36] P. Boucaud, F. de Soto, J.P. Leroy, A. Le Yaounac, J. Micheli, H. Moutarde, O. Pène, and J. Rodriguez-Quintero, Phys. Rev. D 74, 034505 (2006).

[37] P. Boucaud, J.P. Leroy, A. Le Yaounac, A.Y. Lokhov, J. Micheli, O. Pène, J. Rodriguez-Quintero, and C. Roiesnel, JHEP 01, 037 (2006).

[38] F.V. Gubarev, L. Stodolsky, and V.I. Zakharov, Phys. Rev. Lett. 86, 2220 (2001).

[39] F.V. Gubarev and V.I. Zakharov, Phys. Lett. B 501, 28 (2001).

[40] J.A. Gracey, Phys. Lett. B 552, 101 (2003).

[41] D. Dudal, H. Verschelde \& S.P. Sorella, Phys. Lett. B 555, 126 (2003).

[42] D. Dudal, R.F. Sobreiro, S.P. Sorella, and H. Verschelde, Phys. Lett. B 590, 265 (2004).

[43] D. Dudal, R.F. Sobreiro, S.P. Sorella, and H. Verschelde, AIP Conf. Proc. 739, 455 (2005).

[44] K.G. Chetyrkin, S. Narison, and V.I. Zakharov, Nucl. Phys. B 550, 353 (1999).

[45] M.A.L. Capri, V.E.R. Lemes, R.F. Sobreiro, S.P. Sorella, and R. Thibes, Phys. Rev. D 72, 085021 (2005). 